\title{
Community treatment teams in New Zealand - are they suitable for Britain?
}

\author{
Gavin CAPE, Senior Registrar in Psychiatry, Queens Medical Centre, Nottingham; \\ formerly Senior Psychiatric Trainee, Community Mental Health Services, \\ Carrington Hospital, Auckland, New Zealand
}

The run-down of the psychiatric institutions has led to innovative and novel methods of treatment of the mentally ill in the community. In New Zealand, as in other countries, deinstitutionalisation of the more traditional psychiatric services is proceeding rapidly and the statutory and non-statutory community services are straining under the burden. Amid the turmoil of change and the crying out for alternative provisions, a pilot scheme was proposed to serve the mentally ill in the population of West Auckland. In early 1988 the Extended Hours Team (EHT) was born. It is based on the model used in North Sydney, Australia (Hoult, 1986) and Madison, Wisconsin (Stein \& Test, 1980). At the time of conception of the EHT, cost cutting was the rule which led to a difficult gestation but a surprisingly easy delivery and subsequent development over the first year.

The plan was to set up a mobile assessment and treatment team which served the people of West Auckland, a population of approximately 213,000. The catchment area of EHT is also served by an acute psychiatric ward housed in a large Victorian psychiatric hospital which had facilities for rehabilitation, care for the elderly, a specialised drug and alcohol unit, a Maori mental health unit-indeed the full complement of services a traditional asylum is expected to have. This is supplemented by two community mental health centres which provided out-patient care and on-going treatment biased towards a counselling and psychotherapeutic role. One objective in the first six months of operation was to try to establish a decrease in hospital admissions and another was to evaluate the satisfaction level of the consumer and the burden on the relatives. The former objective entailed a comparison of the number of admissions before and after the new service was introduced; the latter was in the form of a consumer survey.

The population is spread out over a large area and has a high proportion of ethnic minorities. Auckland is one of the largest cities in the world by area yet has only a population of just under one million. Approximately $20 \%$ of the population comprise Maori and Polynesian peoples, the rest being of European descent. The area covered and the admixture of cultures posed particular problems in providing a service.

The aims of the service took place in two stages. The first stage was to provide immediate help and treatment to people who are in psychiatric crisis, and to their relatives and social networks, in order to resolve the crisis. The second stage, following on from the first, was to ensure that the person and his/ her relatives are linked to on-going treatment services and have access to further assistance on a 24 hour basis. The target population was those with serious mental illness. Where possible, domiciliary treatment was undertaken as an alternative to hospital treatment. The service was not targeted for those people with a primary diagnosis of drug and alcohol addiction, mental handicap, brain damage or the dementias. If there was doubt about the diagnosis, then an initial assessment was carried out and referral made to another agency if appropriate.

\section{Facilities and staff}

The team was housed on the periphery of the district psychiatric hospital working alongside the PDN (equivalent of the CPN) department. This proximity to a team who served patients with a long-standing illness was often fortuitous as crises or relapses in this group could be seen rapidly and managed in the community when otherwise they would have returned to hospital - thus preventing the career of the 'revolving door patient'.

The EHT operated as a community facility but had close liaison with the general psychiatric hospital. Due to cost and availability of suitable accommodation, the team was housed temporarily in the grounds of the hospital but there were plans to set up base in the heart of the catchment area, away from the asylum. For the service to be effective, accessibility, easy communication, rapid response and the facilities to carry out the necessary assessment and treatment were required. Acceptability and lack of stigmatisation were also seen as important factors.

The service was run by a multidisciplinary team and consisted of a total of ten full-time clinical staff, 
some time of a nurse manager, and a full-time receptionist. The receptionist had the important position of usually being the point of first contact with the patient or referrer as well as sorting out the copious files on patients and community facilities. The clinical team consisted of two medical staff (a consultant and a senior trainee), one social worker, one clinical psychologist and six experienced nursing staff (equivalent of CPN).

The team members were provided with cars, long range pagers and the use of a mobile phone. The range of treatment options was large and included the use of medication, brief family therapy, social systems intervention, and supervision of daily living according to the needs of the patient.

\section{The service}

At present, the team is available for domiciliary visits from 8 a.m. to 11 p.m. (a two shift rota from 8 a.m. to 4 p.m. and 3 p.m. to 11 p.m.) with one person on telephone contact overnight for seven days of the week-hence the name of 'extended hours'. Anyone concerned with or connected with patients (including patients themselves) may contact the service. This was usually by phone, occasionally by visiting the unit. The latter was only allowed in exceptional circumstances, especially at night where staff may be vulnerable. At the time of writing, medical cover at weekends and evenings was being improved with cross cover from other registrars on-call from home.

Domiciliary assessment is essential for the operation of the team. Indeed it allows for a more comprehensive evaluation of patients and their social and living environment and facilitates the build-up of trust and the recruitment of allies necessary to treat the patient (and in many instances the family) effectively without the stigmatisation and labelling of hospital admission. All new referrals were assessed within a few hours of initial contact by two members of different disciplines. The formulation and problem-orientated findings were discussed at the daily team meetings at the beginning of and end of the day shift. It was at these meetings that the level of intervention, and if necessary the appointment of a case manager (synonymous with 'key worker'), was allocated. Brief details of the patient, case manager, diagnosis, and interventions were displayed on a large whiteboard in the office.

There are three levels of intervention provided as a service by EHT to its patients. Firstly case management, where EHT is responsible for formulating and acting on an individual management plan. Following assessment, communication with other parties involved (e.g. family, friends and health and welfare carers) and team discussion, EHT undertakes to be responsible for patients' welfare and health. This is
EHT's most active role where patients (and families) may be visited several times a day if necessary during the acute stages until the patient is seen to improve. It is usual (once the acute episode has resolved) for longer term care or reviews to be required and in these cases it is the responsibility of the team to assertively follow-up the patient, ensuring that transfer of care to another agency occurs-gone are the days of 'lost to follow-up'.

Secondly, there is the support group for patients who may be seen, usually out of normal working hours at the request of another agency who are the primary case managers. This group is seen less intensively and the patient is by and large recovering from a crisis or has a long-standing disorder requiring periods of extra support or supervision. Within the support group are patients who have recently been discharged from the hospital and require a short period of close follow-up.

Lastly there are single contacts where EHT intervenes once or where a referral to a more appropriate service is necessary.

In my experience, there was another function of EHT-a community information resource for individuals and agencies. This became particularly apparent as the service became more established and amassed information on community facilities.

EHT acted in close liaison with an acute admission ward of the district psychiatric hospital, and indeed acted as a filter or gatekeeper for admission to the ward. If possible, EHT would have seen and assessed each of the patients on the ward before admission and, if treatment in the community was not appropriate, would facilitate that person's admission. Frequently early discharge from the hospital was possible with the assistance of initial close follow-up by EHT.

Community Mental Health Centres (CMHCs) in Auckland were biased to seeing patients who were able to work in a psychotherapeutic mode and for those who were recovering from an acute illness requiring longer term management. EHT acted as a filter for these centres and again was in close liaison, often referring to the CMHC once the acute illness had largely resolved.

There were future proposals to move fully into the community and to increase the service and facilities. There was occasional use of two beds in the acute ward for patients requiring a short stay for respite, either for themselves or to give relief to their family. On the whole, this was unsatisfactory. The so-called short-term 'social admission' is poorly catered for in the hospital. One proposal is for the use of supervised short-term respite facilities out of the hospital setting, supervised by EHT staff. Day facilities may also be of benefit. Proposals for increasing the full assessment and treatment service over 24 hours are being examined. 


\section{Evaluation of the service}

Over the first six months of the pilot scheme there were two main aims of evaluation, the effectiveness of the service compared with hospital admission and the level of consumer satisfaction.

The number of new admissions dropped dramatically as compared to the same six month period in each of the previous five years; readmissions were also reduced. The length of time in hospital was on average lower than previous years but this does not seem to have compromised the level of care from a clinical point of view. A small comparison study of clinical outcome measures (using interview rated assessment scales - Brief Psychiatric Rating Scale and the Global Assessment Scale) between EHT case managed patients and hospital in-patients was carried out prospectively. The results from the scores showed that "the community treatment (EHT) group's outcome compares favourably with standard in-patient treatment".

The consumer survey, using an independent researcher, explored the overall satisfaction of patients and the caregivers (i.e. family etc.). Questionnaires took the form of guided interviews, administered by the same interviewer to those patients case managed by the EHT. Acknowledging the bias such surveys may suffer, the conclusions make interesting reading. The patients indicated high levels of satisfaction with the service while the caregivers indicate that the level of burden was greatly reduced. The latter result is of particular interest - the burden change score suggests that rather than community services increasing the level of burden on caregivers, the level of burden is decreased.

\section{Comment}

Why have a community treatment team? What about the experiences of the United States and Italy? What about the burden on relatives (Fadden et al, 1987) and the positive aspects of asylum care (Weller, 1985)? These questions remain only partly answered. The excellent paper by Turner (1988) brings to the discussion table many contentious issues by devotees and critics of community care.

There is a paucity of research on extended hours services and community treatment teams but Stein \& Test (1980) and Hoult (1986) show clinical and social outcomes by this mode of community treatment are as good as, or better than, outcomes achieved by hospital treatment and traditional follow-up. Their work has deficiencies and its critics (Tantam, 1985). Other rationales for CTT are cost based - one-third of costs in hospitals are for hotel services, so community based clinical services enable better use of monies (Mosher, 1983); and not least, clients and relatives prefer community treatment.

This paper suggests that hospital admission is reduced, that community treatment is as good as hospital treatment, clients and their families prefer it, and that there is a reduction in the levels of burden on relatives since the EHT was born in West Auckland. The main problems with the evaluation have been the lack of adequate controls and lack of regard for the ever important cost effectiveness of a new service.

In my experience, working as a psychiatric senior trainee in the EHT, the service provided was excellent. The nature of a domiciliary service provides a far greater insight into the multifactorial aspects of morbidity and an unparalleled experience of crisis intervention, giving me greater understanding of mental illness and its influence on the patient and family.

Dean (1989) describes a home treatment service in a deprived inner city area of Birmingham which runs on similar lines to the West Auckland service - but this is the only British report of its kind. If there is a gradual shift away from a hospital to a community based treatment setting, then has a service such as I have described a place within the British system? I hope so!

\section{Acknowledgement}

I wish to thank all the members of EHT, Dr M. Patton and $\mathrm{Mr} \mathrm{K}$. Brown for their invaluable help. Kia ora.

\section{References}

DeAn, C. (1989) An inner city home treatment service for acute psychiatric patients. Psychiatric Bulletin, 13, 667-669.

Fadden, G., Bebbington, P. \& Kuipers, L. (1987) The burden of care: the impact of functional psychiatric illness on the patient's family. British Journal of Psychiatry, 147, 383-388.

HoulT, J. (1986) Community care of the acutely mentally ill. British Journal of Psychiatry, 149, 137-144.

MOSHER, L. R. (1983) Alternatives to psychiatric hospitalisation: Why has research failed to be translated into practice? New England Journal of Medicine, 309, 1579-1580.

SteIN, L. I. \& TEST, M. A. (1980) Alternative to mental hospital treatment: I. Conceptual model, treatment programme and clinical evaluation. Archives of General Psychiatry, 37, 392-397.

TANTAM, D. (1985) Alternatives to psychiatric hospitalisation. British Journal of Psychiatry, 146, 1-5.

TURNER, T. (1988) Community care. British Journal of Psychiatry, 152, 1-3.

WELLER, M. P. I. (1985) Friern Hospital: where have all the patients gone? The Lancet, $i, 569-571$. 